REVISTAS DE LA FAHCE

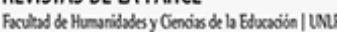

की

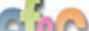

Educación Física y Ciencia

ISSN: 1514-0105

ISSN: 2314-2561

revistaefyc@fahce.unlp.edu.ar

Universidad Nacional de La Plata

Argentina

\title{
Aptidão cardiovascular e coordenação motora apresentam relação com a atenção, flexibilidade cognitiva e velocidade de processamento em crianças de 6 à 10 anos de idade?
}

Mazzoccante, Rafaello Pinheiro; de Luca Corrêa, Hugo; Castro de Sousa, Beatriz Raquel; Castro de Sousa, Ioranny Raquel; Barboza Santos, Marcos Aurélio; Ferreira, Aparecido Pimentel

Aptidão cardiovascular e coordenação motora apresentam relacão com a atenção, flexibilidade cognitiva e velocidade de processamento em crianças de 6 à 10 anos de ídade?

Educación Física y Ciencia, vol. 22, núm. 1, 2020

Universidad Nacional de La Plata, Argentina

Disponible en: http://www.redalyc.org/articulo.oa?id=439963095009

DOI: https://doi.org/10.24215/23142561e117

Esta obra está bajo una Licencia Creative Commons Atribución-NoComercial-Compartirlgual 4.0 Internacional. 


\section{Aptidão cardiovascular e coordenação motora apresentam relação com a atenção, flexibilidade cognitiva e velocidade de processamento em crianças de 6 à 10 anos de idade?}

Are cardiovascular fitness and motor coordination related to attention, cognitive flexibility, and processing speed in children of 6 to 10 years of age?

Rafaello Pinheiro Mazzoccante

Universidade Católica de Brasilia; Centro Universitário

Euro-Americano, UNIEURO, de Brasilia; Centro

Universitário do Distrito Federal., Brasil

rafa_mazzoccante@hotmail.com

Hugo de Luca Corrêa

Universidade Católica de Brasilia; UNIEURO; Centro

Universitário do Distrito Federal., Brasil

Beatriz Raquel Castro de Sousa

Centro Universitário Euro-Americano; Centro

Universitário do Distrito Federal., Brasil

Ioranny Raquel Castro de Sousa

Universidade Católica de Brasilia; Centro Universitário do

Distrito Federal., Brasil

Marcos Aurélio Barboza Santos

Universidade Católica de Brasilia, Brasil

Aparecido Pimentel Ferreira

Universidade Católica de Brasilia, Brasil
DOI: https://doi.org/10.24215/23142561e117

Redalyc: http://www.redalyc.org/articulo.oa?

id $=439963095009$
Recepción: 17 Diciembre 2018

Aprobación: 12 Diciembre 2019

\section{Resumo:}

Objetivo: verificar a relação e a capacidade preditiva do índice de massa corporal, desempenho aeróbio e motor nos diferentes níveis de desempenho da atenção seletiva, flexibilidade cognitiva e velocidade de processamento em crianças. Métodos: trata-se de um estudo transversal realizado em 271 crianças de 6 a 10 anos de idade. Aptidão cardiorrespiratória foi determinada pelo Intermittent Recovery Test - level 1. A coordenação motora foi mensurada pelo teste Körperkoordination für Kinder (KTK), composto por quatro tarefas: trave de equilíbrio, saltos monopedais, transferência sobre plataformas e saltos laterais. Força foi mensurada pelos saltos a $90^{\circ}$ e contramovimento. A atenção e flexibilidade cognitiva foram avaliadas pelo teste de atenção por cancelamento (TAC) (atenção seletiva), teste de trilhas A (atenção sustentada) e B (flexibilidade cognitiva), ambos compostos por tarefa de busca visual. Resultado: as crianças de 6 a 8 anos atingiram maiores valores absolutos nos testes TAC total, Tilhas A soma e Trilhas B (sequencia, conexões e soma) Trilhas B-A em comparação as crianças de de 9 a 11 anos $(\mathrm{p}<0,01)$. Os indicadores de poder de predição do teste Trilhas B e B-A analisando a área sob a curva, com respectivos pontos de corte, foram, índice de massa corporal $=(0,62,<17,90)$ e $(0,62,<17,79)$, escore quociente motor $=(0,81,>63,50)$ e $(0,75,>63,50)$ escore, respectivamente. Conclusão: crianças com maior desempenho aeróbio e motor apresentaram melhores resultados atenção seletiva, atenção sustentada, flexibilidade cognitiva e velocidade de processamento cognitivo em crianças de 6 a 11 anos e estes componentes executivos parecem ser influenciados diretamente pelo IMC e QM, contudo o IMC assemelhou-se de forma negativa.

Palavras-chave: Executive function, Physical fitness, Motor coordination, Selective attencion, Sustained attencion, Cognitive flexibility. 


\begin{abstract}
:
The aim was to verify the relationship and predictive capacity of body mass index, aerobic and motor performance in different levels of selective attention performance, cognitive flexibility and processing speed in children. The method used was a crosssectional study of 271 children aged 6 to 10 . Aerobic fitness was determined by the Intermittent Recovery Test - level 1 . The motor coordination was measured by the Körperkoordination für Kinder (KTK) test, composed of four tasks: balance beam, single-jump jumps, transfer on platforms and lateral jumps. Force was measured by $90^{\circ}$ jumps and countermovement. Cognitive attention and flexibility were assessed by the Attention Cancellation Test (TAC) (selective attention), A (sustained attention) and B (cognitive flexibility), both of which were composed by visual search task. The results show that children aged 6 to 8 reached higher absolute values in the total TAC, Tilhas sum and B tracks (sequence, connections and sum) BA trails compared to children aged 9 to 11 ( $\mathrm{p}$ $<0.01)$. The predictive power indicators of the Tracks B and BA test, which analyze the area under the curve, with respective cutoff points, were body mass index $=(0.62,<17.90)$ and $(0.62,<17,79)$, motor quotient score $=(0.81,>63.50)$ and $(0.75,>63.50)$ score, respectively. We conclude that children with higher motor and aerobic performance presented better results on selective attention, sustained attention, cognitive flexibility and cognitive processing speed in children of 6 to 11 years old.
\end{abstract}

KEYWORDs: Executive function, Physical fitness, Motor coordination, Selective attention, Sustained attention, Cognitive flexibility.

\title{
INTRODUÇão
}

Estudos recentes têm demonstrado haver um crescimento nos déficits de concentração infantil e diminuição da atenção, que por sua vez parece ter implicações diretas no desempenho escolar. Tal constatação tem despertado o interesse de pesquisadores para o desenvolvimento de estratégias direcionadas ao aprimoramento das funções cognitivas com finalidade de melhorar o desempenho escolar, uma vez que o controle cognitivo está relacionado a função executiva (FE) (Budde, Voelcker-Rehage, PietraßykKendziorra, Ribeiro, \& Tidow, 2008; Diamond, 2000; Sigmundsson \& Haga, 2016).

Assim a FE é considerada um elemento essencial no controle e domínio cognitivo, por este componente ser responsável pela capacidade de organização, planejamento, criatividade e autocontrole. Desta forma destacase a FE como uma estrutura fundamental na construção das habilidades cognitivas superiores, esta apresentase como uma medida das funções cognitivas mais aplicável que o próprio quociente de inteligência (Diamond, 2000; Sigmundsson, Englund, \& Haga, 2017).

A FE é dividida em três domínios, sendo eles: a flexibilidade cognitiva (FC), o controle inibitório e a memória de trabalho. A FC é definida como a capacidade de mudar de perspectiva, alterar a maneira de pensar, em função da habilidade de lidar com duas ou mais informações simultaneamente e considerar diferentes abordagens para chegar a solução, sendo portanto, considerada um importante preditor do sucesso escolar e social (Whedon, Perry, Calkins, \& Bell, 2015). Já o controle inibitório é importante para o autocontrole (resistir a tentações) e disciplina (realizar uma tarefa até sua conclusão) e a memória de trabalho é definida como o armazenamento e manipulação temporária da informação, bem como a forma de atuação com certas informações durante a execução de tarefas cognitivas complexas (Diamond, 2014).

Provavelmente todos os componentes do desenvolvimento cognitivo e da FE dependem do controle da atenção (Hazin, et al., 2012), desta forma podemos considerar a atenção como a base para a construção das habilidades cognitivas superiores. Assim, a potencialização do desenvolvimento da cognição infantil parece estar intimamente relacionada à atenção e FE.

O desenvolvimento motor e cognitivo estão inter-relacionados com o crescimento biológico e maturacional, contudo, os desenvolvimentos destes componentes não são necessariamente dependentes da idade cronológica de crianças e adolescentes (Laukkanen, Pesola, Havu, Sääkslahti, \& Finni, 2014). Pesquisas recentes têm apresentado relação positiva entre aptidão cardiorrespiratória e coordenação motora com os componentes da atenção e FC em crianças e adolescentes e a força não apresentou a mesma relação(Mazzoccante et al., 2020; Schmidt et al., 2017). Crianças com melhores índices de aptidão cardiorrespiratória e coordenação motora tendem a apresentar desempenho superior em tarefas escolares 
em comparação às crianças com menores índices, principalmente em tarefas que requerem maior atuação do controle inibitório, FC, processamento rápido da informação, precisão e memória (Afonso, Garganta, \& Mesquita, 2012; Chaddock, Neider, Lutz, Hillman, \& Kramer, 2012; Erickson, Hillman, \& Kramer, 2015; Fernandes et al., 2016; Geertsen et al., 2016; Hillman et al., 2014; Mazzoccante et al., 2020, 2019). Estas descobertas reforçam a importância da aptidão cardiorrespiratória e coordenação motora no desempenho cognitivo e escolar (Geertsen et al., 2016; Laukkanen et al., 2014).

Embora a maioria dos estudos tenham apresentado associação positiva entre a aptidão cardiorrespiratória e coordenação motora como possíveis indicadores na formação da FC e a atenção, dentre outros componentes cognitivos, poucos estudos observaram os efeitos da aptidão cardiorrespiratória, coordenação motora, força e idade cronológica em um único estudo (Schmidt et al., 2017) e nenhum estudo realizado em crianças investigou os indicadores com pontos de cortes determinados pela análise criteriosa da sensibilidade e especificidade como técnica preditora para os componentes da atenção, flexibilidade cognitiva e velocidade de processamento cognitivo, bem como tentar estabelecer um melhor entendimento da relação causa e efeito sobre esse fenômeno. Assim, o objetivo do presente estudo foi verificar a relação entre a aptidão cardiorrespiratória, coordenação motora e força e a capacidade preditiva de seus indicadores de desempenho sobre os níveis de classificação dos componentes da atenção, FC e velocidade de processamento em crianças de 6 a 10 anos.

\section{MÉtodos}

\section{Amostra}

Participaram do estudo 271 estudantes com idades entre 6 a 10 anos (7,53 $\pm 1,52)$, sendo 124 (45,7\%) do sexo masculino e 147 (54,3\%) do sexo feminino, alunos da rede privada de ensino da cidade de Ceilândia, Distrito Federal. A seleção do local de realização e recrutamento dos integrantes do estudo foi realizado por conveniência e todos os participantes tiveram o termo de assentimento e de consentimento livre esclarecido assinados pelos pais ou responsáveis. O estudo foi aprovado pelo Comitê de Ética e Pesquisa da Universidade Católica de Brasília (n².071.564).

\section{Critérios de Inclusão}

Os critérios de inclusão adotados foram a ausência de diagnóstico de patologias neurológicas e/ou psiquiátricas, não apresentar problemas que impedissem a participação nos testes físicos, não estar fazendo uso de medicamentos que alterassem os sentidos ou a cognição e não possuir histórico de reprovação escolar.

\section{Procedimentos gerais}

Os pesquisadores realizaram três visitas para aplicação dos instrumentos de pesquisa, os quais foram realizados de forma randomizada, sendo determinada por sorteio estas visitas, todas realizadas dentro das dependências da escola, divididos em três blocos: 1) teste de atenção por cancelamento e teste de trilhas A e B (aplicados de forma coletiva); 2) testes de saltos e o Intermittent Recovery Test - level 1 (Yo-Yo); 3 ) anamnese, composição corporal e o teste Körperkoordination für Kinder (KTK).

$\mathrm{O}$ ambiente da escola foi adaptado para a realização dos testes e foram realizados pelos mesmos avaliadores. Todos os avaliadores previamente ao início do estudo tiveram duas semanas de instrumentalização dos testes a serem realizados. 


\section{Anamnese}

Foi realizado em formato de entrevista, no qual, o avaliador aplicou um questionário, a fim de assegurar os critérios de inclusão do estudo, como escolaridade, histórico de reprovação escolar e uso de medicamentos.

\section{Teste de trilhas A e B}

O teste de trilhas A é composto por duas folhas, uma contendo letras e outra folha contendo números, ambas dispostas aleatoriamente. O teste consiste em ligar os pontos em ordem alfabética e ordem numérica crescente, respectivamente. Já o teste de trilhas B é composto por uma folha contendo letras e números, dispostos aleatoriamente na mesma folha, no qual a tarefa é ligar letras e números alternadamente nas ordens numéricas e alfabéticas (Montiel \& Capovilla, 2008; Seabra, Alessandra Gotuzo, Dias, 2012). Ambos os testes tiveram seu desempenho estabelecido no tempo máximo de um minuto para cada teste.

Para a análise da trilha $\mathrm{A}$ foi mensurado o número de sequência e na trilha $\mathrm{B}$ foram mensurados sequência, conexões e somatório de ambos (sequências e conexões) (Montiel \& Capovilla, 2008; Seabra, Alessandra Gotuzo, Dias, 2012).

A avaliação de classificação do desempenho no teste de Trilhas A e B foi determinado de acordo com os critérios estabelecidos por Seabra e Dias, 2012 (Seabra, Alessandra Gotuzo, Dias, 2012), no qual, separa os grupos com base no desempenho atingido no teste e o escore categorizado para a idade, separando-os em cinco classificações: muito alta; alta; média; baixa; muito baixa.

\section{Teste de atenção por cancelamento}

Foi aplicado o TAC conforme descrito por Montiel e Capovilla, 2007 (Montiel \& Capovilla, 2008), composto por três partes, com duração máxima de um minuto cada (Seabra, Alessandra Gotuzo, Dias, 2012). Em todos os testes a tarefa do participante é assinalar todos os estímulos iguais ao estímulo-alvo (símbolos) previamente determinado.

$\mathrm{Na}$ primeira parte do teste a figura alvo é indicado na parte superior da folha, aparecendo aleatoriamente 50 vezes e dispostas em 15 linhas em um total de 300 figuras. A segunda parte o estímulo alvo é composto por um par de figuras geométricas que aparece aleatoriamente por 7 vezes, disposta em 15 linhas. Na terceira parte do teste o estímulo-alvo muda e varia de duas a seis vezes a cada linha. Os testes foram avaliados de acordo com o número total de acertos, erros e ausências (Seabra, Alessandra Gotuzo, Dias, 2012).

A avaliação do TAC foi determinado de acordo com os critérios estabelecidos por Seabra e Dias, 2012 (Seabra, Alessandra Gotuzo; Dias, 2012), os quais, separam os grupos com base no desempenho atingido no teste e o escore categorizado para a idade, separando-os em cinco classificações: muito alta; alta; média; baixa; muito baixa.

\section{Avaliação antropométrica}

A avaliação antropométrica e composição corporal foram realizadas pela mensuração da circunferência da cintura e do quadril por meio de uma fita metálica (Sany, TR4010, Brasil), massa corporal por meio de balança eletrônica (Welmy, 6155, Brasil), estatura por estadiômetro (Sanny, ES2040, Brasil) e as dobras cutâneas por adipômetro (Lange, Skinfold Caliper, EUA). Foi estabelecido o índice de massa corporal (IMC = Peso corporal $(\mathrm{kg}) /$ Estatura $^{2}$ metros), índice de conicidade $(\mathrm{IC}=$ Cirfunferência da cintura $(\mathrm{m}) / 0,109 \sqrt{ }$ Peso $(\mathrm{Kg})$ /estatura $(\mathrm{m})$ ) e razão cintura quadril (circunferência da cintura / circunferência do quadril). Para 
porcentagem de gordura $(\% \mathrm{G})$ foi utilizado a equação proposta por Slaughter et al, (Slaughter et al., 2013; Slaughter et al., 1988). As técnicas adotadas para a mensuração das variáveis antropométricas seguiram os procedimentos descritos por Petroski, 1995 (Petroski, Pires-Neto, \& Neto, 1995).

\section{Aptidão cardiorrespiratória}

O teste utilizado foi o Yo-Yo_1, que consiste em correr o máximo de tempo possível em percurso de ida e volta, sendo a distância de 16 metros para crianças de 6 à 9 anos (Ahler, Bendiksen, Krustrup, Wedderkopp, \& George, 2012) e 20 metros acima de 9 anos (Andersen, Andersen, Andersen, \& Anderssen, 2008) e a largura da pista foi de 1,3 metros. Após cada corrida em velocidades progressivamente maiores, intercala-se com um intervalo de descanso de 10 segundos. A progressão da velocidade foi controlada por sons de um CD-player e seu término foi determinado quando o voluntário não conseguiu atingir a marca de 16 ou 20 metros, respectivamente.

Para garantir a realização das corridas nas velocidades correspondentes a cada estágio, um dos avaliadores participava do teste para garantir que o ritmo ideal do teste fosse mantido.

\section{Salto contra movimento e agachado $\left(90^{\circ}\right)$}

A posição inicial de ambos os saltos foi com os pés paralelos na plataforma em uma distância igual a largura dos ombros. Para o salto contra movimento o saltador posicionou-se em pé, fez a flexão dos joelhos, quadris e tornozelos e em seguida estendeu-se verticalmente até saltar sobre o solo. O salto agachado a $90^{\circ}$ o saltador posicionou-se semi-agachado $\left(90^{\circ}\right)$ e em seguida realizou o salto verticalmente sobre o solo estendendo joelhos e quadris. A performace no saltos verticais foi avaliada segundo as descrições do protocolo de Bosco, 1992(Bosco, 2002).

\section{Teste Körperkoordinationstest für Kinder (KTK)}

O KTK é composto por quatro tarefas, 1) trave de equilíbrio: caminhar para trás ao longo de três vigas de largura decrescente, 2) salto monopedais: saltar sobre obstáculos de altura crescente, 3) transferência sobre plataformas: mover-se lateralmente em tábuas de madeira por 20 segundos e 4) saltos laterais: saltar com as duas pernas de um lado para o outro por 15 segundos (Gorla, Araújo, 2009; Hoeboer et al., 2016).

A avaliação da capacidade coordenativa foi realizada pelo quociente motor $(\mathrm{QM})$, oriundo dos valores brutos obtidos em cada tarefa do KTK e seu somatório resultou no QM total, no qual, seus valores estabeleceram os valores do escore do QM total (Gorla, Araújo, 2009).

\section{Análise estatística}

Estatística descritiva com valores de média e desvio padrão foi realizada. A normalidade dos dados foi testada por meio do Shapiro-Wilk test. Para a comparação das variáveis antropométricas, físicas, motoras e congitvas entre as idades utilizou-se o teste t de Student. Para analisar a comparação das variáveis antropométricas, desempenho aeróbio, motor e força frente as diferentes classificações de desempenho dos testes cognitivos, utilizou-se a ANOVA one way, com Post-hoc de Tukey. Para analisar os pontos de corte dos indicadores antropométricos e o desempenho dos testes de força, motor, e aeróbio que pudessem identificar o desempenho nos testes cognitivos, adotou-se a técnica de curva ROC (receiver operating characteristic). A curva ROC foi gerada por meio da plotagem da sensibilidade no eixo y em função de [1 - especificidade] 
no eixo x. O critério utilizado para obtenção dos pontos de corte foram os maiores e mais próximos valores de sensibilidade e especificidade, não inferiores a $60 \%$. A significância estatística de cada análise foi verificada pela área sob a curva ROC e pelo intervalo de confiança a 95\% (IC95\%), de modo que consideramos a habilidade discriminatória significativa quando a área sob a curva ROC estava compreendida entre 1,00 e 0,60 e o limite inferior do IC95\% não inferior a 0,50 . Os modelos de regressão bivariada e multivariada forma utilizados para calcular a razão de chances $(\mathrm{OR})$. O nível de significância do estudo foi $\mathrm{p} \leq 0.05$. O software utilizado foi o Statistical Package for the Social Sciences (SPSS), versão 23.0.

\section{Resultados}

Os dados descritivos referentes à amostra são apresentados na tabela 1 , no qual, as crianças foram separadas em dois grupos determinados pelas idades, sendo o primeiro com as crianças de 6 a 8 anos e o segundo com as crianças de 9 a 10 anos.

TABELA 1

Caracterização da amostra com dados antropométricos, saltos, aptidão cardiorrespiratória e coordenação motora.

\begin{tabular}{|l|c|c|c|}
\hline \multirow{2}{*}{ Variáveis } & 6 a 8 anos & 9 a 10 anos & Valor P \\
\hline Massa corporal (kg) & (Número $=198)$ & (Número $=73$ ) & \\
\hline Estatura (m) & $24,13 \pm 4,91$ & $35,91 \pm 8,36$ & 0,01 \\
\hline IMC (kg-m(-1)) & $1,22 \pm 0,07$ & $1,41 \pm 0,06$ & 0,01 \\
\hline $90^{\circ}$ (cm) & $16,19 \pm 2,18$ & $18,12 \pm 3,48$ & 0,01 \\
\hline Contra movimento(cm) & $17,29 \pm 3,61$ & $20,12 \pm 4,35$ & 0,01 \\
\hline Yo-Yo IR1 performance(m) & $18,24 \pm 4,15$ & $20,35 \pm 4,11$ & 0,01 \\
\hline Trave de equilibrio(escore) & $326,3 \pm 225,7$ & $241,9 \pm 121,1$ & 0,03 \\
\hline Salto monopedal (escore) & $86,88 \pm 10,84$ & $81,92 \pm 11,65$ & 0,01 \\
\hline Salto lateral(escore) & $88,41 \pm 13,43$ & $74,04 \pm 13,51$ & 0,01 \\
\hline Plataforma de transferência(escore) & $90,91 \pm 13,74$ & $84,53 \pm 15,71$ & 0,01 \\
\hline Quocientemotor (escore) & $67,69 \pm 11,38$ & $71,75 \pm 14,43$ & 0,02 \\
\hline TAC Total (soma) & $86,63 \pm 15,78$ & $71,02 \pm 11,47$ & 0,01 \\
\hline Trilhas A soma & $64,39 \pm 16,77$ & $56,06 \pm 18,37$ & 0,01 \\
\hline Trilha B sequencia & $22,81 \pm 2,94$ & $21,84 \pm 2,47$ & 0,01 \\
\hline Trilha B conexões & $10,79 \pm 4,78$ & $6,27 \pm 5,02$ & 0,01 \\
\hline Trilha B soma & $10,24 \pm 4,41$ & $7,09 \pm 4,39$ & 0,01 \\
\hline Trilha B-A & $21,02 \pm 9,06$ & $13,35 \pm 9,06$ & 0,01 \\
\hline
\end{tabular}

IMC = Índice de massa corporal; $\mathrm{QM}=$ quociente motor (escore do somatório dos escores atingidos em cada teste do KTK); TAC = teste de atenção por cancelamento; Trilhas B-A = a diferença entre o teste de trilhas B pelo teste de trilhas A.

Observou-se valores significativamente maiores $(\mathrm{p}<0,05)$ nas variáveis no peso corporal, estatura, IMC, saltos a $90^{\circ}$, contra movimento e plataforma de transferência, para as crianças de 9 a 11 anos em comparação as crianças de 6 a 8 anos. As crianças de 6 a 8 anos obtiveram melhores resultados $(p<0,01)$ em comparação as crianças de 9 a 10 anos, nas seguintes variáveis: a distância percorrida no teste Yo-Yo IR1, trave de equilíbrio, 
salto monopedal, salto lateral, escore do QM, TAC total, Trilhas A soma, Trilhas B (sequência, conexão e soma) e Trilha B-A.

TABELA 2

Apresentam as variáveis da aptidão física, saltos e coordenação motora dentre as diferentes classificações dos testes de atenção.

\begin{tabular}{|c|c|c|c|c|}
\hline \multicolumn{2}{|c|}{ Classificação variáveis } & \multirow{2}{*}{$\frac{\mathrm{N}}{118}$} & \multirow{2}{*}{$\begin{array}{c}\begin{array}{c}\text { Aptidão cardiorrespiratória } \\
\text { (Yo-Yo IR1 performance }(\mathrm{m}) \text { ) }\end{array} \\
336,9 \pm 129,3^{*}\end{array}$} & \multirow{2}{*}{$\begin{array}{c}\mathrm{QM} \\
337,4 \pm 36,2 \mathrm{I}\end{array}$} \\
\hline \multirow{3}{*}{ TAC Total } & Alto e muito alto & & & \\
\hline & Médio & 120 & $289,1 \pm 207,6^{*}$ & $322,9 \pm 31,3^{*}$ \\
\hline & Baixo e muito baixo & 33 & $238,9 \pm 129,3$ & $307 \pm 36,1$ \\
\hline \multirow{3}{*}{ Trilha A } & Alto e muito alto & 28 & $317,3 \pm 279,8$ & $336,9 \pm 29,2$ \\
\hline & Médio & 181 & $311,4 \pm 209,6$ & $326,4 \pm 37,3$ \\
\hline & Baixo e muito baixo & 62 & $274,5 \pm 150,6$ & $325,9 \pm 32,1$ \\
\hline \multirow{3}{*}{ Trilha B } & Alto e muito alto & 103 & $312,5 \pm 193,2$ & $335,1 \pm 36,3 \geq ¥$ \\
\hline & Médio & 148 & $302,5 \pm 220,4$ & $322,6 \pm 34,5$ \\
\hline & Baixo e muito baixo & 12 & $244,9 \pm 110,6$ & $332,7 \pm 31,5$ \\
\hline \multirow{3}{*}{ Trilha B-A } & Alto e muito alto & 95 & $304,8 \pm 186,8$ & $334,9 \pm 36,2 ¥$ \\
\hline & Médio & 144 & $315,2 \pm 225,5$ & $323,3 \pm 35,4$ \\
\hline & Baixo e muito baixo & 24 & $228,5 \pm 142,7$ & $328,8 \pm 29,2$ \\
\hline
\end{tabular}

$\mathrm{QM}=$ Quociente motor (somatório dos escores atingidos em cada teste do KTK); TAC $=$ Teste de atenção por cancelamento; ${ }^{*} \mathrm{p}<0,05$ em relação a classificação baixo e muito baixo; ${ }^{* *} \mathrm{p}<0,01$ em relação a classificação baixo e muito baixo; $¥ \mathrm{p}<0,05$ em relação a classificação médio; $¥ ¥ \mathrm{p}<0,01$ em relação a classificação médio; \# p <0,01 em relação a classificação baixo, muito baixo e médio.

A tabela 2 apresenta os resultados de desempenho no teste Yo-Yo IR1 e somatório do QM, de acordo com classificação dos testes de atenção, flexibilidade cognitiva e velocidade de processamento cognitivo. Como observado na tabela 2, os valores de QM de crianças classificadas como alto e muito alto foram maiores quando comparados com as crianças classificadas como baixo e muito baixo nos testes, TAC total, tilhas B, trilhas B-A $(p<0,05)$. Adicionalmente, observa-se que os valores desempenho no teste Yo-Yo IR1 das crianças classificas como alto e muito alto para o TAC total foi significativamente maior que as crianças classificadas como baixo e muito baixo.

TABELA 3

Área sob a curva ROC e IC95\%, ponto de corte, sensibilidade e especificidade e odss ratio, entre indicadores antropométricos, de desempenho aeróbio, motor e força com a atenção seletiva e sustentada.

\begin{tabular}{|c|c|c|c|c|c|}
\hline \multicolumn{2}{|r|}{ Variáveis } & Área sob a curva & $\begin{array}{l}\text { Ponto de } \\
\text { corte }\end{array}$ & $\begin{array}{l}\text { Sensibilidade/ } \\
\text { especificidade }\end{array}$ & Odss Ratio \\
\hline \multirow{5}{*}{ 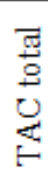 } & $\mathrm{IMC}\left(\mathrm{kg} \cdot \mathrm{m}^{2(-1)}\right)$ & $0,62(0,55-0,69)$ & $<18,65$ & $(0,88-0,80)$ & $0,67(0,37-1,20)$ \\
\hline & Yo-Yo IR1 performance (m) & $0,60(0,53-0,67)$ & $>124$ & $(0,94,-0,86)$ & $2,80(1,09-7,23)^{*}$ \\
\hline & Salto $90^{\circ}(\mathrm{cm})$ & $0,33(0,26-0,41)$ & & Não preditivo & \\
\hline & Salto contra movimento $(\mathrm{cm})$ & $0,30(0,23-0,36)$ & & Não preditivo & \\
\hline & Quociente motor (escore) & $0,66(0,59-0,73)$ & $>63,50$ & $(0,89-0,81)$ & $1,60(0,66-3,89)$ \\
\hline \multirow{5}{*}{ 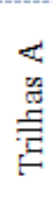 } & $\operatorname{IMC}\left(\mathrm{kg} \cdot \mathrm{m}^{2(-1)}\right)$ & $0,62(0,55-0,69)$ & & Não preditivo & \\
\hline & Yo-Yo IR1 performance (m) & $0,47(0,35-0,58)$ & & Não preditivo & \\
\hline & Salto $90^{\circ}(\mathrm{cm})$ & $0,58(0,48-0,68)$ & & Não preditivo & \\
\hline & Salto contra movimento $(\mathrm{cm})$ & $0,51(0,39-0,62)$ & & Não preditivo & \\
\hline & Quociente motor (escore) & $0,49(0,39-0,58)$ & & Não preditivo & \\
\hline
\end{tabular}


${ }^{*}$ Valores significativos de acordo com o IC.

Ao analisar os indicadores antropométricos e desempenhos de força, motor e cardiorrespiratório (tabela 3). Somente apresentaram preditores significativos do desempenho da atenção seletiva (IC $\geq 0,50$; área $\geq$ 0,60) o IMC, desempenho no Yo-Yo IR1, escore do QM, nenhum indicador mostrou-se preditor da atenção sustentada (trilhas A). Adicionalmente os resultados do OR, somente o desempenho do Yo-Yo IR1 (>124 $\mathrm{m}$ ) apresentou 2,8 vezes mais chances de alcançarem a classificação de desempenho alto e muito alto referente no teste TAC.

\section{TABELA 4}

Área sob a curva ROC e IC95\%, ponto de corte, sensibilidade e especificidade e odss ratio, entre indicadores antropométricos, de desempenho aeróbio, motor e força com a flexibilidade cognitiva e velocidade de processamento cognitivo.

\begin{tabular}{|c|c|c|c|c|c|}
\hline \multicolumn{2}{|r|}{ Variáveis } & Área sob a curva & $\begin{array}{l}\text { Ponto de } \\
\text { corte }\end{array}$ & $\begin{array}{l}\text { Sensibilidade/ } \\
\text { especificidade }\end{array}$ & Odss Ratio \\
\hline \multirow{5}{*}{ 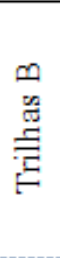 } & $\operatorname{IMC}\left(\mathrm{kg} \cdot \mathrm{m}^{2(-1)}\right)$ & $0,62(0,55-0,69)$ & $<17,90$ & $(0,85-0,72)$ & $0,45(0,23-0,89)^{*}$ \\
\hline & Yo-Yo IR1 performance (m) & $0,55(0,48-0,62)$ & & Não preditivo & \\
\hline & Salto $90^{\circ}(\mathrm{cm})$ & $0,35(0,29-0,42)$ & & Não preditivo & \\
\hline & Salto contra movimento $(\mathrm{cm})$ & $0,32(0,25-0,38)$ & & Não preditivo & \\
\hline & Quociente motor (escore) & $0,81(0,75-0,86)$ & $>63,50$ & $(0,93-0,82)$ & $2,91(1,22-6,91)^{*}$ \\
\hline \multirow{5}{*}{ 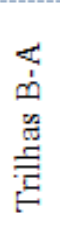 } & $\operatorname{IMC}\left(\mathrm{kg} \cdot \mathrm{m}^{2(-1)}\right)$ & $0,62(0,55-0,69)$ & $<17,79$ & $(0,84-0,73)$ & $0,48(0,25-0,90)^{*}$ \\
\hline & Yo-Yo IR1 performance $(\mathrm{m})$ & $0,53(0,46-0,60)$ & & Não preditivo & \\
\hline & Salto $90^{\circ}(\mathrm{cm})$ & $0,37(0,30-0,43)$ & & Não preditivo & \\
\hline & Salto contra movimento $(\mathrm{cm})$ & $0,37(0,30-0,44)$ & & Não preditivo & \\
\hline & Quociente motor (escore) & $0,75(0,69-0,82)$ & $>63,5$ & $(0,95-0,81)$ & $4,35(1,63-11,6)^{*}$ \\
\hline
\end{tabular}

${ }^{*}$ Valores significativos de acordo com o IC.

Os indicadores antropométricos, de desempenho de força, motor e aeróbio, somente apresentaram como preditores significativos de flexibilidade cognitiva e velocidade de processamento cognitivos (IC $\geq 0,50$; área $\geq 0,60$ ) o IMC e o escore do QM, resultados apresentados na tabela 4.

Adicionalmente os resultados do odss ratio, apresentaram maiores chances de obterem a classificação de desempenho alto e muito alto na flexibilidade cognitiva e velocidade de processamento cognitivos as crianças que alcançaram valores: IMC $(<17,90 ;<17,79)$ e o escore do $\mathrm{QM}>63,50$, respectivamente (tabela 4). Representando a razão de chances de 52 á 55\% para o IMC e para o escore de QM de 2,91 e 4,35 vezes mais chances de alcançarem a classificação de desempenho alto e muito alto no teste de trilhas B e trilhas BA, respectivamente (tabela 4 ).

\section{Discussão}

Os principais resultados do presente estudo apresentaram a influência direta de crianças com o IMC abaixo de 17,79 á 18,65, valores estes dentro da classificação eutrófica, obtiveram maiores chances de atingem maior desempenho de $33 \%$ para a atenção seletiva, de $55 \%$ para a flexibilidade cognitiva e de $52 \%$ para a velocidade de processamento. As crianças com maior desempenho aeróbio demonstraram uma chance de 2,8 vezes maior de atingirem maior capacidade de atenção seletiva, agora as crianças com maior desempenho motor mostraram-se uma maior probabilidade de 1,6 vezes para atenção seletiva, de 2,91 vezes para a flexibilidade cognitiva e 4,35 vezes para a velocidade de processamento. Assim destaca-se as crianças com melhor desempenho de atenção seletiva, flexibilidade cognitiva e velocidade de processamento, apresentaram melhor coordenação motora, e outro fator relevante que a idade cronologica não mostrou-se um componente 
determinante dos resultados no desempenho da aptidão cardiorrespiratória, coordenação motora, atenção, flexibilidade e velocidade de processamento cognitivo. Somente os indicadores de força expresso pelo saltos não apresentaram relação com nenhum componente da função executiva.

Corroborando os resultados do presente estudo, alguns autores observaram o comportamento das variáveis aptidão cardiorrespiratória, coordenação motora fina e grossa, domínios cognitivos e desempenho acadêmico em crianças de 7 a 10 anos e constataram associação positiva entre o desempenho motor e aeróbio com melhores respostas de memória de trabalho, memória semântica, atenção sustentada, velocidade de processamento cognitivo, capacidade de aprendizagem, desempenho acadêmico em matemática e compreensão leitora, desta forma, destaca-se uma relação entre maior atividade elétrica cerebral, controle inibitório, FC, precisão e memória com maior aptidão cardiorrespiratória e motora (Chaddock et al., 2012; Erickson et al., 2015; Geertsen et al., 2016; Mazzoccante et al., 2020, 2019; Schmidt et al., 2017), a força de impulsão não apresentou relação com estes componentes destacados, resultados vão em concordância aos encontrados pelo estudo.

A coordenação motora, apresenta forte capacidade de influenciar a FC e atenção. Nesse sentido, uma revisão sistemática analisou a relação entre a habilidade motora e cognitiva em estudos com crianças e adolescentes de 4 a 16 anos e encontraram dados que confirmam essa relação (van der Fels et al., 2015). Os autores acreditam que há uma maior ativação do componente da atenção em atividades que exigem coordenação motora finas, bilateral e agilidade, demonstrando a necessidade do componente cognitivo durante a realização de uma tarefa motora complexa (van der Fels et al., 2015).

A relação entre a aptidão cardiorrespiratória, coordenação motora com a FC, atenção sustentada e velocidade de processamento verificadas no presente estudo, pode ser explicada pelos estímulos sociais, físicos, motores e cognitivos vivenciados na vida pregressa dessa criança, uma vez que quanto maior o nível de atividade física desta a criança, melhor será seus domínios atencionais e executivos. Reforçando essa hipótese, um estudo verificou uma melhora na capacidade de atenção sustentada e foco em adolescentes de 13 a 16 anos após uma única sessão exercícios de coordenação motora complexa (Budde et al., 2008). Essa relação enfatiza a importância da estimulação motora como uma estratégia para a melhora da FC e atenção, variáveis que apresentam grande relação com o sucesso escolar e social (Diamond, 2012; Diamond \& Lee, 2011). O aumento nos valores da coordenação motora pode estar relacionado com o aumento dos componentes da atenção, flexibilidade cognitiva, velocidade de processamento e a medida de FE.

O desenvolvimento e crescimento motor e cognitivo ocorrem concomitantemente e de forma integrada com a maturação. A ativação destes dois componentes são estritamente associadas, já que os circuitos neurais recrutados durante a ação motora pelo cerebelo, também atuam nos circuitos neurais responsáveis pela atenção e FE, achados reafirmados pelos indicadores de predição da coordenação motora com a FC e velocidade de processamento (tabela 4). $\mathrm{O}$ exercício físico parece ser uma estratégia eficiente para o aperfeiçoamento motor, aeróbio, atencional e da FC. Para alguns autores, os possíveis mecanismos que podem explicar esse fenomeno está atrelada as mudanças funcionais e neuro estruturais propiciadas pelo aumento da aptidão cardiorrespiratória, como: angiogenese, aumento na quatidade de neurotransmissores, aumento fluxo sanguíneo cerebral e em especial, maior ativação do córtex pré-frontal decorrentes de tarefas motoras novas e/ou complexas (Diamond, 2000; Fernandes et al., 2016; Geertsen et al., 2016; Mazzoccante et al., 2020; Sigmundsson \& Haga, 2016). Crianças com maior desempenho cardiorrespiratória e motor podem apresentar melhores desempenhos na atenção seletiva, flexibilidade cognitiva e velocidade de processamento cognitivo em comparação as crianças com menor desempenho cardiorrespiratória e motor, afirmações demonstradas pelos resultados do estudo.

Entendemos como uma limitação dos resultados do referido estudo, a condição de não podermos definir os principais mecanismos neurofisiológicos factíveis pelos resultados encontrados, por tratar-se de um estudo transversal, não nos permite o estabelecimento de causa-efeito. Outra limitação está referida aos testes utilizados não avaliarem a atividade neural de maneira direta, todavia, são testes capazes de mensurar, indicar 
e discriminar os diferentes domínios da atenção e FC, uma vez que são validados para crianças e adolescentes, com ampla aplicação em diversos estudos (DIAS \& SEABRA, 2017; Hillman et al., 2014; Mazzoccante et al., 2020, 2019; Menezes, Dias, Trevisan, Carreiro, \& Seabra, 2015; van der Niet et al., 2016). Ademais, são de fácil aplicação, boa acurácia e reprodutibilidade, portanto, ideais para serem utilizados no ambiente escolar (Hazin, Falcão, et al., 2012).

Apesar das limitaçóes descritas acima, acredita-se que o presente estudo pode trazer uma contribuição para essa temática, particularmente apresentando uma das aplicabilidades que ficaram evidenciadas com os resultados, uma vez que parece fundamental a necessidade de se aprimorar a capacidade cardiorrespiratória e a coordenação motora de crianças e adolescentes a fim de melhorar a performance e desenvolvimento cognitivo. Adicionalmente, destaca-se a necessidade de que mais estudos possam investigar a relação da aptidão cardiovascular e coordenação motora sobre a atenção e flexibilidade cognitiva em crianças, particularmente apresentando indicadores do desempenho aeróbio e motor atrelados a valores desejados em relação a atenção seletiva, sustentada, flexibilidade cognitiva e velocidade de processamento cognitivo.

Conclui-se que o IMC aptidão cardiorrespiratória e coordenação motora parecem estar relacionadas com o desempenho dos componentes da função executiva, uma vez que, as crianças com maior desempenho aeróbio e motor e menor IMC apresentaram melhores resultados atenção seletiva, atenção sustentada, flexibilidade cognitiva e velocidade de processamento cognitivo, ademais, a competências cardiorrespiratória, motora e o IMC foram apontadas como indicadores interessantes para a promoção dos componentes da FE.

\section{Agradecimientos}

A fundação de Apoio à Pesquisa do Distrito Federal, pela concessão de bolsa de iniciação científica.

\section{REFERÊNCIAS}

Afonso, J., Garganta, J., \& Mesquita, I. (2012). A tomada de decisão no desporto: o papel da atenção, da antecipação e da memória. Revista Brasileira de Cineantropometria \& Desempenho Humano, 592-601. https://doi.org/10. 5007/1980-0037.2012v14n5p592

Ahler, T., Bendiksen, M., Krustrup, P., Wedderkopp, N., \& George, K. P. (2012). Aerobic fitness testing in 6- to 9year-old children: Reliability and validity of a modified Yo-Yo IR1 test and the Andersen test. European Journal of Applied Physiology, 112(3), 871-876. https://doi.org/10.1007/s00421-011-2039-4

Andersen, L. B., Andersen, T. E., Andersen, E., \& Anderssen, S. A. (2008). An intermittent running test to estimate maximal oxygen uptake: The Andersen test. Journal of Sports Medicine and Physical Fitness, 48(4), 434-437.

Bosco, C. (2002). La valutazione della forza con il test di Bosco. Societá Stampa Sportiva (2nd ed.). Roma.

Budde, H., Voelcker-Rehage, C., Pietraßyk-Kendziorra, S., Ribeiro, P., \& Tidow, G. (2008). Acute coordinative exercise improves attentional performance in adolescents. Neuroscience Letters, 441, 219-223. https://doi.org/ 10.1016/j.neulet.2008.06.024

Chaddock, L., Neider, M. B., Lutz, A., Hillman, C. H., \& Kramer, A. F. (2012). Role of childhood aerobic fitness in successful street crossing. Medicine and Science in Sports and Exercise, 44(4), 749-753. https://doi.org/10.124 9/MSS.0b013e31823a90cb

Diamond, A. (2000). Close Interrelation of Motor Development and Cognitive Development and of the Cerebellum and Prefrontal Cortex. Child Development, 71(1), 44-56. https://doi.org/10.1111/1467-8624.00117

Diamond, A. (2012). Activities and Programs That Improve Children's Executive Functions. Current Directions in Psychological Science, 21(5), 335-341. https://doi.org/10.1177/0963721412453722

Diamond, A. (2014). Executive Functions. Annual Review of Clinical PsychologyPsychol., 64, 135-168. https://doi.o $\mathrm{rg} / 10.1146 /$ annurev-psych-113011-143750.Executive 
Diamond, A., \& Lee, K. (2011). Interventions shown to aid executive function development in children 4 to 12 years old. Science, 333(6045), 959-964. https://doi.org/10.1126/science.1204529

DIAS, N. M., \& SEABRA, A. G. (2017). School performance at the end of elementary school: Contributions of intelligence, language, and executive functions. Estudos de Psicologia (Campinas), 34(2), 315-326. https://doi. org/10.1590/1982-02752017000200012

Erickson, K. I., Hillman, C. H., \& Kramer, A. F. (2015). Physical activity, brain, and cognition. Current Opinion in Behavioral Sciences, 4, 27-32.

Fernandes, V. R., Michelle L. Scipião Ribeiro, T. M., Maciel-Pinheiro, P. de T., Guimarães, T. T., Araújo, N. B., Ribeiro, S., \& Deslandes, A. C. (2016). Motor coordination correlates with academic achievement and cognitive function in children. Frontiers in Psychology, 7(MAR), 318. https://doi.org/10.3389/fpsyg.2016.00318

Geertsen, S. S., Thomas, R., Larsen, M. N., Dahn, I. M., Andersen, J. N., Krause-Jensen, M., ... Lundbye-Jensen, J. (2016). Motor skills and exercise capacity are associated with objective measures of cognitive functions and academic performance in preadolescent children. PLoS ONE, 11(8), 1-16. https://doi.org/10.1371/journal.p one. 0161960

Gorla, JI, Araújo PF, R. J. (2009). Avaliação motora em educação física adaptada: teste ktk. (3rd ed.). Sao Paulo: Phorte.

Hazin, I., Falcão, J. T. D. R., Gomes, E., Garcia, D., Samantha, R. C., Menezes, M. T., \& Dias, M. D. G. B. B. (2012). Dados Normativos do Teste de Atenção por Cancelamento em Estudantes do Ensino Fundamental. Psico, 43, $428-436$.

Hazin, I., Tarcísio, J., Falcão, R., Garcia, D., Gomes, E., Cortez, R., ... Dias, B. (2012). Dados Normativos do Teste de Atenção por Cancelamento (TAC) em Estudantes do Ensino Fundamental. Psico, 43(4), 428-436.

Hillman, C. H., Pontifex, M. B., Castelli, D. M., Khan, N. A., Raine, L. B., Scudder, M. R., ... Kamijo, K. (2014). Effects of the FITKids Randomized Controlled Trial on Executive Control and Brain Function. Pediatrics, 134(4), e1063-e1071. https://doi.org/10.1542/peds.2013-3219

Hoeboer, J., De Vries, S., Krijger-Hombergen, M., Wormhoudt, R., Drent, A., Krabben, K., \& Savelsbergh, G. (2016). Validity of an Athletic Skills Track among 6- to 12-year-old children. Journal of Sports Sciences, 34(21), 20952105. https://doi.org/10.1080/02640414.2016.1151920

Laukkanen, A., Pesola, A., Havu, M., Sääkslahti, A., \& Finni, T. (2014). Relationship between habitual physical activity and gross motor skills is multifaceted in 5- to 8-year-old children. Scandinavian Journal of Medicine \& Science in Sports, 24(2), e102-e110. https://doi.org/10.1111/sms.12116

Mazzoccante, R. P., Corrêa, H. D. E. L., Santana, F. S. D. E., Câmara, M. A., Raquel, B., Sousa, C. D. E., ... Melo, G. F. D. E. (2020). Attention and executive function are PREDICTED BY ANTHEROPOMETRIC INDICATORS, STRENGTH , MOTOR PERFORMANCE, AND AEROBIC FITNESS IN CHILDREN AGED 6 - 10 YEARS, Human Movement21(1), 40-48.

Mazzoccante, R. P., Corrêa, H. D. L., Queiroz, J. L. De, Sousa, R. C. De, Raquel, I., Sousa, C. De, \& Barboza, M. A. (2019). The relationship of sports practice with motor performance, selective attention, cognitive flexibility and processing speed in children aged 7 to 10 years, J Hum Dev, 29(September 2018), 365-372.

Menezes, A., Dias, N. M., Trevisan, B. T., Carreiro, L. R. R., \& Seabra, A. G. (2015). Intervençãoo para funções executivas no transtorno do deficit de atenção e hiperatividade. Arquivos de Neuro-Psiquiatria, 73(3), 227-236. https://doi.org/10.1590/0004-282X20140225

Montiel, josé maria, \& Capovilla, alessandra gotuzo seabra. (2008). Teste de Atenção por Cancelamento\#: análise de critérios de correção. Cognição, 54, 289-296.

Petroski, E. E. L., Pires-Neto, C. S., \& Neto, C. P. (1995). Validação de Equações Antropométricas para estimativa da densidade Corporal em Mulheres. Revista Brasileira de Atividade Física e Saúde.

Schmidt, M., Egger, F., Benzing, V., Jäger, K., Conzelmann, A., Roebers, C. M., \& Pesce, C. (2017). Disentangling the relationship between children's motor ability, executive function and academic achievement. PLoS ONE, 12(8). https://doi.org/10.1371/journal.pone.0182845

Seabra, Alessandra Gotuzo; Dias, N. M. (2012). Avaliação neuropsicológica cognitiva: atenção e funções executivas. $\operatorname{MEMNON}$ (Vol. 1). 
Sigmundsson, H., Englund, K., \& Haga, M. (2017). Associations of Physical Fitness and Motor Competence With Reading Skills in 9- and 12-Year-Old Children: A Longitudinal Study. SAGE Open, 7(2). https://doi.org/10. $1177 / 2158244017712769$

Sigmundsson, H., \& Haga, M. (2016). Motor competence is associated with physical fitness in four- to six-year-old preschool children. European Early Childhood Education Research Journal, 24(3), 477-488. https://doi.org/1 0.1080/1350293X.2016.1164411

Slaughter, A. M. H., Lohman, T. G., Boileau, R. A., Horswill, C. A., Stillman, R. J., Loan, M. D. V. A. N., \& Bemben, D. A. (2013). Skinfold Equations for Estimation of Body Fatness in Children and Youth Published by\#: Wayne State University Press Stable URL\#: http://www.jstor.org/stable/41464064. Human Biology, 60(5), 709-723.

Slaughter, M., Lhoman, T., Boileau, R., Horswill, C., Stillman, R., Van Loan, M., \& Bemden, D. (1988). Slaughter, M. H., Skinfold Equations for Estimation of Body Fatness in Children and Youth , Human Biology, 60:5 (1988:Oct.) p.709. Children, 60(5), 709-723.

van der Fels, I. M. J., te Wierike, S. C. M., Hartman, E., Elferink-Gemser, M. T., Smith, J., \& Visscher, C. (2015). The relationship between motor skills and cognitive skills in 4-16 year old typically developing children: A systematic review. Journal of Science and Medicine in Sport, 18(6), 697-703. https://doi.org/10.1016/j.jsams.2014.09.007

van der Niet, A. G., Smith, J., Oosterlaan, J., Scherder, E. J. A., Hartman, E., \& Visscher, C. (2016). Effects of a Cognitively Demanding Aerobic Intervention during Recess on Children's Physical Fitness and Executive Functioning. Pediatric Exercise Science, 28(1), 64-70. https://doi.org/10.1123/pes.2015-0084

Whedon, M., Perry, N. B., Calkins, S. D., \& Bell, M. A. (2015). Changes in Frontal EEG Coherence across Infancy Predict Cognitive Abilities at Age 3: The Mediating Role of Attentional Control. Dev Psychol, 52(9), 11731178. https://doi.org/10.1126/science.1249098.Sleep

\section{BY-NC-SA}

